\title{
Using query reformulation to compare learning behaviors in Web search engines
}

\author{
Marcelo Tibau \\ Federal University of the State of Rio \\ de Janeiro \\ Rio de Janeiro, Brazil \\ e-mail: marcelo.tibau@uniriotec.br
}

\author{
Sean W. M. Siqueira \\ Federal University of the State of Rio \\ de Janeiro \\ Rio de Janeiro, Brazil \\ e-mail: sean@uniriotec.br
}

\author{
Bernardo Pereira Nunes \\ Federal University of the State of Rio \\ de Janeiro / Pontifical Catholic \\ University of Rio de Janeiro \\ Rio de Janeiro, Brazil \\ e-mail: bnunes@inf.puc-rio.br \\ Ruben Francisco Manrique \\ Universidad de los Andes \\ Bogotá, Colombia \\ e-mail:rf.manrique@uniandes.edu.co
}

\begin{abstract}
Web search engines have gained importance as tools capable of connecting informal and self-learning with formal learning by aiding individuals in retrieving relevant information through the formulation and modification of their queries. Understand the differences between query states and their transitions becomes increasingly important, as doing so makes the optimization of search engines' results according to educational uses and needs possible. This paper introduces the ESKiP Taxonomy of Query States, a classification framework validated in an experiment involving two different query log datasets. It enables the comparison between the behaviors of users in search for knowledge (learners) and users performing transactional or factual searches in Web search engines.
\end{abstract}

Query reformulation; query states; searching as learning; Web search engine; exploratory search; knowledge-intensive process

\section{INTRODUCTION}

World Wide Web (Web) is the most popular medium for accessing the Internet and has been central to the development of the data-information-centric environment we live in today. Search engines are one of the most frequently used tools for discovering and retrieving information on the Web [10, 11], the analysis of Web search patterns has become a frequent issue in investigations centered on user interaction with Information Retrieval systems [5, 10, 14].

Exploratory search, the specialization of information seeking that combines activities of querying and browsing in order to obtain information [16] has emerged as one of the fundamental skills in an environment that has digital technology as one of its main driving forces [9]. Its exploratory nature derives from the fact that users engage in complex cognitive tasks focused on explorations of retrieved information, which may lead to learning and knowledge acquisition or the development of (intellectual) skills [16]. Learning from the search process, through the search activities performed, is a concept present in Searching as Learning [13]. The development of searching systems that could support critical and creative learning through search behavior is one of the main topics of its research agenda. Rieh et al. [13] cite evaluating the usefulness of information critically, differentiating resources, monitoring results, tracking information, prioritizing actions and applying sense-making as examples of these types of behaviors.

At the intersection of exploratory search and the vastness of available data lie the concepts of Searching as Learning, which also open up the possibility of using Web search engines as tools to support learning. While examining the searching process, the concept of query states can be used to successfully model the sequence of human-machine interactions that take place during a search $[2,6]$. The state of a query is based on the set of terms applied to consecutive queries within a search session, and is determined by classes of behavior, such as uniqueness, generalization, specialization and reformulation.

Query reformulation, as defined by Jansen, Booth and Spink [6], is the process of altering a given query to improve search or retrieval performance. As a research subject, its importance arises from the indication that effective query reformulation can enhance the outcome of a user's searches [4], as it directly correlates to the amount of information needed to complete a search task [7]. As more query reformulations are issued during searching, the cognitive effort spent in more detailed explorations and the available information increase. Liu et al. [7] determined a percentage of $47.19 \%$ in effective reformulation, meaning that more than half of users' attempts to refine the search results are noneffective, thus inspiring our aim of improving the identification of Query States, and their transitions.

The research presented in this paper is based on the concept of Exploratory Search as a Knowledge-intensive Process (also known as KiP) [8, 15]. It explores the query reformulation possibilities provided by the informationgathering behavior embedded in an exploratory search as a $\mathrm{KiP}$ and its application to compare the behaviors of users in search for knowledge (i.e., learners), and users performing transactional or factual searches in Web search engines.

To properly identify the types of query reformulation prompted by the information gathered in search activities and the knowledge process it generates, it was necessary to consider some specific behavioral patterns KiP brought to exploratory search, such as subject familiarization, which 
improves learner's capacity to select a search term, as well as the abilities to control the search process itself, and to assess the relevance of the retrieved information. To map the transitions of query states as learners acquire new knowledge regarding their search topic (which, in turn, they do by reformulating their queries), a taxonomy was proposed: the ESKiP Taxonomy of Query States (hence "Taxonomy").

To validate the Taxonomy, two query logs datasets were used to outline the frequency of query reformulation types. In the ensuing log analysis, the Taxonomy was observed to help point out different search strategies applied by learners and users in the datasets.

In order to report on our research process and findings, the paper has been organized as follows: Section 2 reviews existing literature; Section 3 presents the Taxonomy and addresses its complexity. Section 4 explains the Taxonomy's evaluation setup. Finally, Section 5 presents the results.

\section{RELATED WORK}

Research focusing on users conceiving and modifying their queries during a session tends to rely on query reformulation. Rieh and Xie [12] conducted a qualitative analysis, reporting on three facets of query reformulation content, format, and resource - with sub-facets related to each of the three areas. Özmutlu and Çavdur [10] investigated the use of neural networks for automatically identifying topic changes for queries within sessions, and defined a terminology and sub-groups that echoed in subsequent studies $[3,6,7]$. They defined 'topic' as a group of queries submitted by a single user on a single topic and 'session' as a group of queries submitted by a single user.

Jansen, Booth and Spink [6] later updated the definition of session to "a series of queries submitted by a user and related interactions during an episode of interaction between the user and the Web search engine around a single topic", adding a temporal character.

The implementation of a KiP perspective to the process of modelling search interactions indicates that user can - and often do - change the topic of a search during a session $[8$, 15]. This might be due to the user's lack of sufficient information, leaving them unable to adequately define the search problem or solution at the early stages of a search, a characteristic of exploratory searches. In this case, the research presented in this paper updates both Özmutlu and Çavdur's [10] and Jansen, Booth and Spink's [6] incomplete reference to search "around a single topic" by considering search interactions around not only single but also multiple topics. The formalization provided by the Taxonomy additionally adds specificity to Jansen, Booth and Spink's [6] generic way of dealing with the definition of a session (as "related interactions during an episode of interaction") by asserting its search interactions within a given period.

Liu et al. [7] created a taxonomy of query reformulation with five categories representing different reformulation types: Generalization (G), Specialization (SC), Repeat (RP), Word Substitution (WS) and New (N). The authors represented the first and second compared queries as Qi and Qi+1 respectively, Qi+1 being the query immediately following the query Qi in the same session.

The ESKiP Taxonomy deals with the use of syntactically different but semantically related terms, a case not covered by Liu et al.'s taxonomy. The authors cited the possibility of 'New' terms being related to the previous queries instead of being different ones, for example, by being composed of acronyms or synonyms. The specific problem concerned the classification of the query reformulation type 'New' and was handled by the introduction of the Related State (RE) in the ESKiP Taxonomy.

Another innovation introduced by the ESKiP Taxonomy is the definition of an initial search state as a reformulation type, which was not a priority for previous studies on query reformulation $[3,6,7,10,12]$, but is an important one to the present research due to the appearance of a recurring situation: the return to an initial state after specializations. A demonstration of such case is the following search sequence: 'lake como' -> lake como vacation season' -> 'lake como'$>$ 'lake como rental prices'. Consider the set $\mathrm{Q}$ of the previous sequence as (q1, q2, q3, q4), and note that $\mathrm{q} 3$ represents a return to the initial state $\mathrm{q} 1$.

The "Term Criterion Labels" presented at Exploratory Search KiP model's Decision Diagram [8, 15] (and used to represent users' decision-making pattern on results checking, query formulation, and modification activities) was not enough to account for all query reformulation possibilities. For instance, the labels $\mathrm{K} 1, \mathrm{~K} 2, \mathrm{~K} 3$ and $\mathrm{K} 4{ }^{1}$ depicted there could not adequately differentiate the initial states of distinct search sessions presented by an Exploratory Search Episode.

Neither represented transitions that were more precisely refined, and could not be accounted as specializations and generalizations, such as the modification of certain words within a term without changing the query length. They also found situations such as the ones displayed by the semantically related terms case scenario, and the return to the initial state query term during the same search session (the 'lake como' example presented above), challenging.

The Query States presented in the ESKiP Taxonomy update the Exploratory Search KiP model regarding its query reformulation's representational fragility.

\section{A QUERY REFORMULATION TAXONOMY}

The complexity of a query reformulation taxonomy is reflected by the reasoning and human expressions it can represent. This diversity is per se a major challenge and one, which increases when important questions assessing the user's educational need (when considering a Web search engine a learning tool) are added. Are query states and transitions connected to learners/users search strategies? Could learner/user search strategies be used to identify search profiles? Could search profiles be used to set retrieval rankings?

To begin answering these questions, the ESKiP Taxonomy required a number of formalizations necessary to adequately

${ }^{1} \mathrm{~K} 1$ (General term); K2 (Specific term related to K1); K3 (Specific term not related to $\mathrm{K} 1)$; $\mathrm{K} 4$ (Term not related to subject). 
represent the knowledge a query reformulation induces. This formalization required not only subjects composing the action of Web search, such as a query and a session, but also those concerned to user behavior. An example is the definition of search profiles, which, although not direct connected to query states, is important to the information retrieval used to optimize search engines' results.

The following formalizations were defined:

- Term: a series of keywords or characters used within a search engine and separated by white space or other type of separator.

- Query: a string of terms submitted by a user, combining all the texts, numbers and symbols entered into a search engine.

- Session: a group of queries submitted by a user during an event or a group of events of interaction between the user and the search engine around a single or multiple topics within a given period.

- Exploratory Search Episode: one or more sessions conducted by the same individual user within a given period.

- Query Reformulation: the process of altering a given query to improve search or retrieval performance.

- Query States: set of terms used to reformulate queries within a session, determined by a user's decisionmaking process regarding whether to include, exclude, modify or keep the set of terms. Also referred to in this work as "query reformulation types".

- User Search Strategies: relating to behavioral attributes associated with a user's decision-making process that determines the way in which he or she decides to transition from one query state to another within a session, or an exploratory search episode by including, excluding, modifying or keeping the chosen set of terms in order to refine the search engine's responses.

- Search Profiles: a set of implemented user search strategies, which determine a Web search pattern.

A collaboration between Australian, Brazilian and Colombian universities, the ESKiP Taxonomy is an adaptation of the existing classification of query reformulation originally created by Liu et al. [7], added to by an initial state of a search and the return to the initial state after specialization. The related reformulation type was also added. The Taxonomy is present in Table I.

TABLE I. ESKIP TAXONOMY OF QUERY STATES

\begin{tabular}{|l|l|l|}
\hline \multicolumn{1}{|c|}{ Query State } & \multicolumn{1}{c|}{ Definition } & \multicolumn{1}{c|}{ Examples } \\
\hline Initial State (IS) & $\begin{array}{l}\text { Qi contains at least one term and } \\
\text { represents the start of a search. }\end{array}$ & Lake Como \\
\hline $\begin{array}{l}\text { Return State } \\
\text { (RS) }\end{array}$ & $\begin{array}{l}\text { Qi contains at least one term and } \\
\text { represents the start of a search or } \\
\text { a previous search query; Qi+n }\end{array}$ & $\begin{array}{l}\text { Lake Como -> } \\
\text { Lake Como } \\
\text { Vacation -> } \\
\text { Lake Como }\end{array}$ \\
\hline
\end{tabular}

${ }^{2}$ LearnWeb is a collaborative system that provides advanced features for searching, organizing and sharing learning. It can be accessed at

\begin{tabular}{|c|c|c|}
\hline Query State & Definition & Examples \\
\hline & $\begin{array}{l}\text { contains exactly the same term } \\
\text { of Qi. }\end{array}$ & \\
\hline $\begin{array}{l}\text { Generalization } \\
\text { (GE) }\end{array}$ & $\begin{array}{l}\text { Qi and Qi+1 have at least one } \\
\text { term in common; Qi+1 contains } \\
\text { fewer terms than Qi. }\end{array}$ & $\begin{array}{l}\text { Brazilian Flag } \\
\text { Colors -> } \\
\text { Brazilian Flag }\end{array}$ \\
\hline $\begin{array}{l}\text { Specialization } \\
\text { (SC) }\end{array}$ & $\begin{array}{l}\text { Qi and Qi+1 have at least one } \\
\text { term in common; Qi+1 contains } \\
\text { more terms than Qi. }\end{array}$ & $\begin{array}{l}\text { Brazilian Flag } \\
->\quad \text { Brazilian } \\
\text { Flag Colors }\end{array}$ \\
\hline Repeat (RP) & $\begin{array}{l}\text { Qi and Qi+1 may contain } \\
\text { exactly the same terms, but the } \\
\text { format of these terms may be } \\
\text { different. }\end{array}$ & $\begin{array}{l}\text { Brazilian Flag } \\
->\quad \text { Flag } \\
\text { Brazilian }\end{array}$ \\
\hline $\begin{array}{l}\text { Word } \\
\text { Substitution } \\
\text { (WS) }\end{array}$ & $\begin{array}{l}\text { Qi and Qi+1 have at least one } \\
\text { term in common; Qi+1 might } \\
\text { has the same length as Qi, but } \\
\text { contains some terms that are not } \\
\text { in Qi. }\end{array}$ & $\begin{array}{l}\text { Brazilian Flag } \\
->\text { Colombian } \\
\text { Flag }\end{array}$ \\
\hline New (NW) & $\begin{array}{l}\text { Qi and Qi+1 do not have any } \\
\text { common terms. }\end{array}$ & \begin{tabular}{l}
\multicolumn{2}{c}{ Brazilian Flag } \\
$->\quad$ Dog \\
Breeds
\end{tabular} \\
\hline Related (RE) & $\begin{array}{l}\text { Qi and Qi+1 do not have any } \\
\text { common terms, but have similar } \\
\text { or related meaning. }\end{array}$ & $\begin{array}{l}\text { The Girl with } \\
\text { the Dragon } \\
\text { Tattoo } \\
\text { Actress -> } \\
\text { Rooney Mara } \\
\text { images }\end{array}$ \\
\hline
\end{tabular}

Another addition to the definition is the role assumed by the letter Q, which represents a query. Qi and Qi+1 have the same definition as depicted by Liu et al. [7]. Qi+n represents a query used during the same search session as Qi, but not the query immediately following, a necessity introduced by the Return State (RS).

\section{EVALUATION SETUP}

To evaluate the ESKiP Taxonomy, it was necessary to verify its representational performance. For this experiment, a dataset (the Learn Web dataset) was composed by selecting sessions and queries collected from transaction logs of an online student-teacher the LearnWeb-OER ${ }^{2}$ professional community which has an embedded search engine tool. The only modification done to the data was the changing of the original alphanumeric session ID to a more easily identified session name (e.g. LearnWeb01, LearnWeb02). The Learn Web dataset is comprised of 646 queries divided into 159 sessions [15].

The proposed Query States were manually identified in the Learn Web dataset to examine the usage of the Taxonomy's query reformulation types (Fig.1) and to determine its representational adequacy by validating the epistemic requirement of Knowledge Representation Schemes evaluation. Bingi, Khazanchi and Yadav [1] define the requirement, asserting that the representation must provide the ability to express the facts it wishes to express. The presence and commonness of each proposed Query State was verified. 


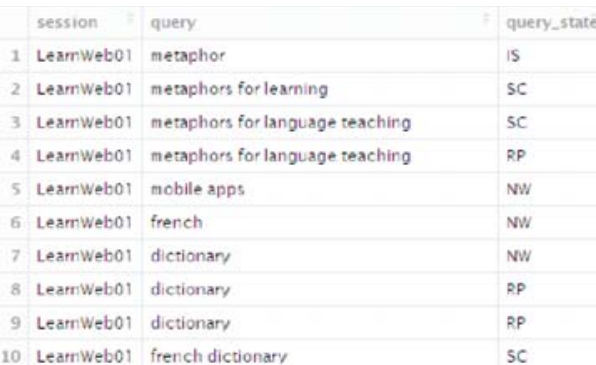

Figure 1. A sample from Learn Web dataset.

To observe their presence and commonness, the frequency of each query reformulation type was counted. Table II shows their frequency at the Learn Web dataset.

TABLE II. QUERY STATES IDENTIFIED AT LEARN WEB DATASET

\begin{tabular}{|l|l|l|}
\hline \multicolumn{1}{|c|}{ Query State } & \multicolumn{1}{|c|}{ Frequency } & \multicolumn{1}{c|}{$\begin{array}{c}\text { Overall } \\
\text { Frequency }\end{array}$} \\
\hline Initial State (IS) & 159 & $24.61 \%$ \\
\hline Return State (RS) & 8 & $1.24 \%$ \\
\hline Generalization (GE) & 17 & $2.63 \%$ \\
\hline Specialization (SC) & 40 & $6.19 \%$ \\
\hline Repeat (RP) & 278 & $43.03 \%$ \\
\hline Word Substitution (WS) & 17 & $2.63 \%$ \\
\hline New (NW) & 83 & $12.85 \%$ \\
\hline Related (RE) & 44 & $6.81 \%$ \\
\hline
\end{tabular}

To validate the Taxonomy and to verify the consistency of query reformulation types, it was necessary to corroborate the appearance of all proposed Query States in a different set of Exploratory Search Episodes. For this purpose, a Yahoo! ${ }^{3}$ Dataset, comprising of queries taken from Yahoo US Web Search and from TREC Session track (2010-2013) ${ }^{4}$ (Fig.2), was used. The sessions were demarcated using a 30 minute time-out and comprised queries made in natural and free search conditions. In this sense, the Yahoo! Dataset is more generic and has a larger scope. The Query States were manually identified, and the eight proposed query reformulation types had their frequency mapped, as seen in Table III.
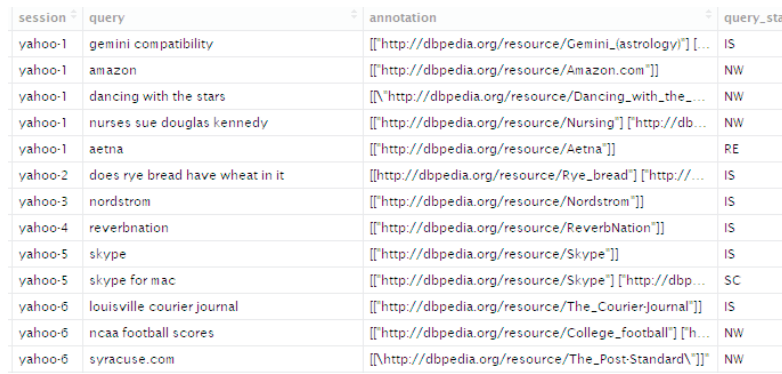

Figure 2. A sample from Yahoo! dataset.

\footnotetext{
${ }^{3}$ Yahoo! Webscope dataset ydata-search-query-log-to-entities-v1_0 [http://labs.yahoo.com/Academic_Relations]

${ }^{4}$ With combining 2633 queries in 845 sessions, 7482 spans, and 5964 links
}

TABLE III. QUERY STATES IDENTIFIED AT YAHOO! DATASET

\begin{tabular}{|l|l|l|}
\hline \multicolumn{1}{|c|}{ Query State } & \multicolumn{1}{|c|}{ Frequency } & \multicolumn{1}{c|}{$\begin{array}{c}\text { Overall } \\
\text { Frequency }\end{array}$} \\
\hline Initial State (IS) & 845 & $32.09 \%$ \\
\hline Return State (RS) & 6 & $0.23 \%$ \\
\hline Generalization (GE) & 91 & $3.46 \%$ \\
\hline Specialization (SC) & 324 & $12.31 \%$ \\
\hline Repeat (RP) & 79 & $3.00 \%$ \\
\hline Word Substitution (WS) & 529 & $20.09 \%$ \\
\hline New (NW) & 551 & $20.93 \%$ \\
\hline Related (RE) & 208 & $7.90 \%$ \\
\hline
\end{tabular}

An observable result is the appearance of each Query State in both datasets. The Initial State (IS) marks the start of a session and, as expected, accounts for the number of each session present in the datasets. The overall frequency of query reformulation types does however indicate the presence of different Search Profiles within the datasets. The overall frequency was calculated by using the number of queries present in each dataset to determine the percentage of each Query State by the frequency of its appearance (e.g. in Learn Web dataset SC appeared 40 times from 646 queries, hence an overall frequency of $6.19 \%$ ).

Rha, Shi and Belkin [3] defined specialization as the most frequent information reason to reformulate. In the Yahoo! Dataset, specialization was responsible for $12.31 \%$ of query reformulations, while in the Learn Web dataset it accounted for a mere 6.19\%. The most frequent Query State present in the Learn Web dataset is a RP, a choice made in $43.03 \%$ of the cases. This means that users preferred to repeat the same query several times in a row. In the Yahoo! dataset, the most frequent reason to reformulate was to start a NW query in the same session $(20.93 \%)^{5}$.

\section{RESULTS}

Since users' intentions can only be speculated upon at present - the data did not provide such information - based on the data's origins it seems plausible that search intention did play a role in defining learners and users' search strategies. The Learn Web dataset originated from a collaborative search and sharing system, which links different online services and provides advanced support for organizing and sharing the resources found online. By comparison to the Yahoo! dataset, the Learn Web contains searches that demonstrate a more exploratory pattern, in which the information available needs to be explored in more detail. The search engine logs supplied by Yahoo! reflect a less exploratory pattern - and maybe explain the reason behind the user's behavior in changing search subjects so often during the same session.

WS accounted for $20.09 \%$ of queries reformulation in the Yahoo! and for $2.63 \%$ in the Learn Web dataset, corroborating the effect caused by the data's origin. A User Search Strategy based on WS indicates a tendency to refine information retrieval by finding the words that could prompt the best results, e.g. q1 = 'yahoo mail india' and q2 = 'yahoo mail plus'

\footnotetext{
to Wikipedia articles.

${ }^{5}$ Note that Initial State (IS) could not be considered a reason to reformulate because it is a mandatory initial Query State.
} 
- taken from Yahoo! dataset. The substitution can also be between words that have a semantic relation, such as $\mathrm{q} 1=$ 'Easter egg chase' and q2 = 'Easter egg hunt'. Note that in both examples, the queries' length was maintained as three words, and there are two common words, 'yahoo mail' and 'Easter egg'.

The Learn Web dataset illustrates a preference for adding more terms to the query $(6.19 \%)$ or finding related terms (6.81\%) over the other approaches, since the SC and RE Query States are the most frequent choices of search refinement, neither of which entirely changes the subject but rather indicates a greater awareness about the searched topics than the WS strategy. A predilection for a term (RP) in consecutive queries $(43.03 \%)$ was also observed. This indicates that users may want to revisit information or even find or check for new information on topics they have previously explored. This information might be employed, for example, to determined context differences based on User Search Strategies and to assign a learning purpose to the performed searches.

The identification of terms' sequences by the classification of query reformulation types and their labeling as Query States aid the visualization of the search strategies learners/users adopt. It can be used to define Search Profiles based on learners/users' search shrewdness and applied to profiles to design search interfaces that more adequately support learning with respect to the search process. Examples of potential applications include terms suggestions for the composition of queries, tips to aid the user improve in search abilities, and recommended search paths. These findings indicate that Query States can be considered to identify query reformulation and to map learners/users search behaviors, thus contributing to advance research agendas regarding Information Retrieval and Searching as Learning.

The added three states (IS, RS and RE) was a necessity derived by this research's goal to identify the informationgathering behavior embedded in an exploratory search as a KiP. However, the Taxonomy has the potential to add more finer-grained semantic. Given recent advances in Natural Language Processing (i.e., word and sentence embedding and automatic linguistic analysis tools), similar additions are not only welcomed but required to explore how far apart two query reformulations are.

Finally, the ESKiP Taxonomy opens more opportunities in the field of knowledge representation and reasoning in helping to represent the structure and behavior of queries reformulation in search systems.

\section{ACKNOWLEDGMENT}

This study was financed in part by the 'National Council for Scientific and Technological Development (CNPq) Brazil' - Process 315374/2018-7, Project 'Searching as Learning: the information search as a tool for learning.

\section{REFERENCES}

[1] Bingi, R., Khazanchi, D., \& Yadav, S. B. (1995). A framework for the comparative analysis and evaluation of knowledge representation schemes. Information Processing and Management, 31(2), 233-247.
[2] Choo, C., Detlor, B., \&Turnbull, D. (1998).A behavioral model of information seeking on the Web: Preliminary results of a study of how managers and it specialists use the Web. In C.M. Preston (Ed.), Proceedings of the 61st Annual Meeting of the American Society for Information Science (ASIS) (pp. 290-302). Medford, NJ: Information Today.

[3] EY Rha, W Shi, NJ Belkin. (2017). An exploration of reasons for query reformulations. Proceedings of the Association for Information Science and Technology, Wiley Online Library, Volume54, Issue1. Pages 337-346.

[4] Gauch, S., \& Smith, J. (1993). An expert system for automatic query reformulation. Journal of the American Society for Information Science, 44(3), 124-136.

[5] Ingwersen, P. (1996). Cognitive perspectives of information retrieval interaction: elements of a cognitive IR theory. Journal of Documentation, 52(1), 3-50.

[6] Jansen, B.J., Booth, D.L., Spink, A.: Patterns of query reformulation during Web searching. J. Assoc. Inf. Sci. Technol. 60(7), 1358-1371 (2009)

[7] Liu, C., Gwizdka, J., Liu, J., Xu, T., \& Belkin, N. J. (2010). Analysis and evaluation of query reformulations in different task types. Proceedings of the American Society for Information Science and Technology, 47(1), 1-9.ACM.

[8] M. Tibau, S. W. M. Siqueira, B. Pereira Nunes, M. Bortoluzzi and I. Marenzi, Modeling Exploratory Search as a Knowledge-Intensive Process. 2018 IEEE 18th International Conference on Advanced Learning Technologies (ICALT), Mumbai, 2018, pp. 34-38.

[9] Maynard, A. D. Navigating the fourth industrial revolution. Nature Nanotechnology, 10(12), 1005-1006. Nature Publishing Group. http://dx.doi.org/10.1038/nnano.2015.286. 2015.

[10] Özmutlu, H.C., \& Çavdur, F. (2005). Application of automatic topic identification on Excite Web search engine data logs. Information Processing \& Management, 41(5), 1243-1262.

[11] Özmutlu, S., Özmutlu, H. C., \& Spink, A. (2004). A day in the life of Web searching: an exploratory study. Information Processing and Management, 40(2), 319-345.

[12] Rieh, S.Y., \& Xie, H. (2006). Analysis of multiple query reformulations on the web: The interactive information retrieval context. Information Processing \& Management, 42(3), 751-768.

[13] Rieh, S. Y., Collins-Thompson, K., Hansen, P., \& Lee, H.-J. Towards searching as a learning process: A review of current perspectives and future directions. Journal of Information Science, 42(1), 19-34. https://doi.org/10.1177/0165551515615841. 2016.

[14] Spink, A. (1998). Toward a theoretical framework for information retrieval (IR) within an information seeking context. In Proceedings of the 2nd international information seeking in context conference, 12-15 August 1998, Sheffield. UK: University of Sheffield, Department of Information Studies.

[15] Tibau M., W. M. Siqueira S., Pereira Nunes B., Bortoluzzi M., Marenzi I., Kemkes P. (2018) Investigating Users' Decision-Making Process While Searching Online and Their Shortcuts Towards Understanding. In: Hancke G., Spaniol M., Osathanunkul K., Unankard S., Klamma R. (eds) Advances in Web-Based Learning - ICWL 2018. ICWL 2018. Lecture Notes in Computer Science, vol 11007. Springer, Cham.

[16] White, Ryen W, and Roth, Resa A. Exploratory search: beyond the query-response paradigm. Synthesis lectures on information concepts, retrieval, and services, 1(1): 1-98. Print. 2009. 